\title{
Las terapias psicológicas podrían reducir la depresión y ansiedad en las mujeres víctimas de violencia de pareja
}

\author{
Psychological therapies could reduce depression and anxiety in women victims of intimate partner violence
}

\section{Comentado de:}

Hameed M, et al. Psychological therapies for women who experience intimate partner violence. Cochrane Database Syst Rev. 2020 Jul 1;7(7):CD013017. PMID:32608505 ${ }^{1}$

\section{Objetivos}

Evaluar la efectividad de las terapias psicológicas para las mujeres que sufren violencia de pareja (VDP).

\section{Desenlaces principales}

Se consideraron como desenlaces primarios la depresión, la autoeficacia y un indicador de efecto perjudicial (abandonos) en el seguimiento de seis a 12 meses. Entre los resultados secundarios se evaluaron otros síntomas de salud mental, ansiedad, calidad de vida, reexposición a VDP, planificación de la seguridad y comportamientos, el uso de la atención sanitaria y de los servicios de VDP, y el apoyo social.

\section{Métodos de búsqueda}

Se realizaron búsquedas en el registro de ensayos controlados del Grupo Cochrane de Trastornos Mentales Comunes (CCMDCTR, por sus iniciales en inglés), CENTRAL, MEDLINE, Embase, CINAHL, PsycINFO y en otras tres bases de datos, hasta finales de octubre de 2019. También se realizaron búsquedas en los registros de ensayos internacionales para identificar ensayos no publicados o en curso, y búsquedas manuales en revistas seleccionadas, en las listas de referencias de los ensayos incluidos y en la literatura gris.

\section{Criterios de selección}

Se incluyeron ensayos controlados aleatorizados (ECA), cuasialeatorizados, ECA grupales y ensayos cruzados (cross-over) de terapias psicológicas, con mujeres de 16 años 0 más que informaron por sí mismas sobre su experiencia reciente o durante la vida de VDP. Se incluyeron los ensayos si las mujeres también experimentaban diagnósticos de problemas de salud mental coexistentes, de abuso de sustancias o ambos. Las terapias psicológicas incluían una amplia gama de intervenciones dirigidas a la cognición, la motivación y el comportamiento, en comparación con la atención habitual, ningún tratamiento, o intervenciones aplazadas o mínimas. Se clasificaron las terapias psicológicas de acuerdo con la lista de terapias psicológicas del Grupo Cochrane de Trastornos Mentales Comunes.

\section{Obtención y análisis de los datos}

Dos autores de la revisión extrajeron los datos y realizaron la evaluación del riesgo de sesgo. Se compararon los efectos del tratamiento entre las intervenciones experimentales y las de comparación a corto plazo (hasta seis meses después de la valoración inicial), a mediano plazo (entre seis y menos de 12 meses, punto temporal del resultado primario) y el seguimiento a largo plazo (12 meses y más). Se utilizó la diferencia de medias estandarizada (DME) para los resultados continuos y el odds ratio (OR) para los resultados dicotómicos, y se realizaron meta-análisis de efectos aleatorios, debido a la alta heterogeneidad entre los ensayos.

\section{Resultados principales}

Se incluyeron 33 ensayos de terapias psicológicas en los que participaron 5.517 mujeres asignadas al azar a intervenciones experimentales (2.798 mujeres, $51 \%$ ) y de comparación $(2.719$ mujeres, $49 \%$ ).

Las terapias psicológicas incluían 11 terapias integradoras, nueve terapias humanísticas, seis terapias cognitivoconductuales (TCC), cuatro TCC de tercera ola y otras tres intervenciones de orientación psicológica. No hubo ensayos clasificados como terapias psicodinámicas.

La mayoría de los ensayos se realizaron en países de altos ingresos (19 en los EE.UU., tres en Irán, dos en Australia y dos en Grecia, y un ensayo en China, España, India, Kenya, Nigeria, Pakistán y el Reino Unido), con mujeres reclutadas en centros de atención sanitaria, comunitarios, en contextos de acogida o de refugio, o una combinación de cualquiera de ellos.

Las terapias psicológicas se realizaron en su mayor parte cara a cara (28 ensayos), pero variaron en cuanto a la duración del tratamiento (de dos a 50 sesiones) y al personal que las administraba (trabajadores sociales, enfermeras, psicólogos, trabajadores sanitarios de la comunidad, médicos de familia, investigadores).

El tamaño medio de la muestra fue de 82 mujeres (14 a 479), con una edad media de 37 años, y el $66 \%$ estaba sin empleo. La mitad de las mujeres estaba casada o vivía con una pareja y poco más de la mitad de las participantes había experimentado VDP en los últimos 12 meses (17 ensayos), el $6 \%$ en los últimos dos años (dos ensayos) y el $42 \%$ durante su vida (14 ensayos).

Mientras que 20 ensayos (61\%) describieron estrategias fiables de muestreo aleatorio de bajo riesgo, sólo 12 ensayos (36\%) describieron procedimientos fiables para ocultar la asignación del estado de la participante.

Mientras que 19 ensayos midieron la depresión de las mujeres, sólo cuatro ensayos midieron la depresión como un resultado continuo en el seguimiento a medio plazo. Estos mostraron un probable efecto beneficioso de las terapias psicológicas en la reducción de la depresión (DME -0,24; Intervalo de Confianza [IC] del 95\%: -0,47 a -0,01; cuatro ensayos, 600 mujeres evidencia de certeza moderada). Sin embargo, para la autoeficacia, puede no haber evidencia de una diferencia entre los grupos (DME -0,12; IC del $95 \%$ : -0,33 a 0,09; un ensayo con datos de seguimiento a medio plazo, 346 mujeres; evidencia de baja certeza). Además, es posible que no haya diferencias entre el número de mujeres que abandonaron los grupos de intervención experimental o de comparación, un indicador de ausencia de efectos perjudiciales (OR 1,04, IC del 95\%: 0,75 a 1,44; cinco ensayos con datos de seguimiento a medio plazo, 840 mujeres; evidencia de certeza baja). Aunque ningún ensayo informó sobre los eventos adversos de las terapias psicológicas o la participación en el ensayo, sólo un ensayo midió los resultados de los efectos perjudiciales utilizando una escala validada. En cuanto a los resultados secundarios, los ensayos midieron la ansiedad sólo en el seguimiento a corto plazo, lo que demuestra que las terapias psicológicas pueden reducir los síntomas de la ansiedad (DME -0,96; IC del 95\%: -1,29 a -0,63; cuatro ensayos, 158 mujeres; evidencia de certeza baja). Sin embargo, dentro del seguimiento a medio plazo, la evidencia de certeza baja reveló que puede no haber evidencia entre los grupos para los resultados de planificación de la seguridad (DME 0,04; IC del 95\%: -0,18 a 0,25; un ensayo, 337 mujeres), el trastorno de estrés postraumático (DME -0,24; IC del $95 \%$ : -0,54 a 0,06; cuatro ensayos, 484 mujeres) o la reexposición a cualquier forma de VDP (DME 0,03; IC del 95\%: -0,14 a 


\section{0,2; dos ensayos, 547 mujeres).}

\section{Conclusiones de los autores}

Hay evidencia de que para las mujeres que experimentan VDP, las terapias psicológicas probablemente reducen la depresión y pueden reducir la ansiedad. Sin embargo, no se sabe con certeza si las terapias psicológicas mejoran otros resultados (autoeficacia, trastorno de estrés postraumático, reexposición a la VDP, planificación de la seguridad) y hay datos limitados sobre los efectos perjudiciales. Por lo tanto, si bien las terapias psicológicas probablemente mejoran la salud emocional, no está claro si este enfoque aborda las necesidades continuas de las mujeres en materia de seguridad, apoyo y curación holística de los traumas complejos. Se necesitan más intervenciones centradas en enfoques de trauma y ensayos más rigurosos (con resultados consistentes en puntos temporales de seguimiento similares), ya que no se pudo sintetizar gran parte de la investigación.

Fuentes de financiamiento/Conflicto de interés de los autores: Universidad de Melbourne y Australian General Practice Training, Australia, proporcionaron financiamiento a uno de los autores de la revisión. El autor principal declaró no tener conflictos de interés. Otros autores de la revisión declararon haber participado en algunos de los ensayos incluidos, lo que podría representar mayor conocimiento sobre esos estudios; se minimizó este inconveniente evitando que los investigadores extrajeran datos o valoraran el riesgo de sesgo de los trabajos en los que estuvieron involucrados.

\section{Comentario}

El impacto en la salud integral de las personas víctimas de violencia por razones de género ${ }^{2}$ es tan amplio como las diversas modalidades en las que se expresa. Conceptualizar ese impacto sobre el proceso salud/enfermedad/atención implica poder entender a este tipo de violencia como un factor de riesgo o vulnerabilidad ${ }^{3}$. El abordaje requiere de dispositivos interdisciplinarios e intersectoriales ${ }^{4}$ que contemplen los determinantes sociales de la salud y puedan escapar de la lógica patologizante. La violencia de género (VDG) tiene una lógica compleja, rara vez tiene una única etiología y sus sobrevivientes no necesariamente padecen una patología instalada. Lejos de la medicalización, la intervención efectiva requiere comprender sus mitos fundantes, funcionamiento y condicionantes, incorporando una perspectiva de género y Derechos Humanos. La elección del enfoque de intervención debería poder considerar a la persona como alguien que se encuentra atravesando consecuencias subjetivas esperables de la vulneración de sus derechos más básicos, evitando la patologización, la revictimización y la iatrogenia.

Esta revisión Cochrane considera el abandono del tratamiento como un efecto adverso. Sin embargo la VDG se presenta con una modalidad cíclica que comprende diferentes etapas (crisis, arrepentimiento y acumulación de tensión) ${ }^{5}$. La ruta crítica que deben iniciar las personas para salir de esta situación se caracteriza por múltiples avances y retrocesos íntimamente relacionados con este ciclo. El tratamiento integral debe contemplar estas particularidades para no leer erróneamente los retrocesos o abandonos de tratamiento como un efecto adverso, sino como insertos en una lógica de subordinación mucho más amplia que impide la toma de decisiones desvinculadas de la misma.

El artículo señala que las terapias psicológicas fueron administradas por diferentes actores (trabajadores sociales, enfermeras, psicólogos, trabajadores sanitarios de la comunidad, médicos de familia, investigadores). Resulta importante diferenciar el tratamiento psicológico llevado adelante por profesionales de la salud matriculados, de los acompañamientos a víctimas de violencia de género que podrían realizar otras personas. Si bien se encontró evidencia de que algunas terapias psicológicas probablemente reducen la depresión y pueden reducir los síntomas de ansiedad, no han demostrado su efectividad en otras sintomatologías o trastornos asociados a la exposición a la violencia. Sería recomendable incluir en futuras investigaciones, trabajos que evalúen los resultados de intervenciones interdisciplinarias en violencia de género.

\section{Conclusiones de las comentadoras}

Consideramos fundamental pensar la VDG como un ciclo dinámico, donde los avances y retrocesos son esperables en el proceso de acompañamiento a una persona que intenta salir de esa situación de vulnerabilidad. Interpretar el abandono del tratamiento como un efecto adverso podría ser incorrecto en este contexto.

Resultan necesarios más estudios que contemplen la complejidad del abordaje de esta problemática. Sería deseable para futuras investigaciones la incorporación de un enfoque centrado en la interdisciplina, que incorpore la perspectiva de género y derechos humanos, como elementos necesarios para evaluar los efectos de las intervenciones.

Ximena García Bianchi [ Servicio de Medicina Familiar y Comunitaria, Hospital Italiano de Buenos Aires; Comité para el seguimiento de familias con problemáticas complejas en el Bajo Boulogne, Centro de Medicina Familiar y Comunitaria San Pantaleón. ximena.garcia@hospitalitaliano.org.ar ]

Adriana Beatriz Goldman [ Servicio de Medicina Familiar y Comunitaria, Hospital Italiano de Buenos Aires; Comité para el seguimiento de familias con problemáticas complejas en el Bajo Boulogne, Centro de Medicina Familiar y Comunitaria San Pantaleón. adriana.goldman@hospitalitaliano.org.ar ]

Ivana Carballeira [ Comité para el seguimiento de familias con problemáticas complejas en el Bajo Boulogne, Centro de Medicina Familiar y Comunitaria San Pantaleón. ivana.carballeira69@gmail.com ]

Melisa Eberling [ Servicio de Medicina Familiar y Comunitaria, Hospital Italiano de Buenos Aires; Comité para el seguimiento de familias con problemáticas complejas en el Bajo Boulogne, Centro de Medicina Familiar y Comunitaria San Pantaleón; Departamento de Salud Pública, Instituto Universitario Hospital Italiano de Buenos Aires. melisa.elberling@hospitalitaliano.org.ar ] 
Carolina Guerra [ Servicio de Medicina Familiar y Comunitaria, Hospital Italiano de Buenos Aires; Comité para el seguimiento de familias con problemáticas complejas en el Bajo Boulogne, Centro de Medicina Familiar y Comunitaria San Pantaleón. carolina.guerra@hospitalitaliano.org.ar ]

García Bianchi X, Goldman A, Caballeira I, Elberling M, Guerra C. Las terapias psicológicas podrían reducir la depresión y ansiedad en las mujeres víctimas de violencia de pareja. Evid Actual Pract Ambul. 2020;23(4):e002095. Comentado de: Hameed M, et al. Psychological therapies for women who experience intimate partner violence. Cochrane Database Syst Rev. 2020 Jul 1;7(7):CD013017. PMID:32608505

\section{Referencias}

1. Hameed M, O'Doherty L, Gilchrist G, et al. Psychological therapies for women who experience intimate partner violence. Cochrane Database Syst Rev. 2020;7(7):CD013017. Available from: 10.1002/14651858.CD013017.pub2.

2. Argentina. Ministerio de Justicia y Derechos Humanos. Ley 26485 de protección integral para prevenir, sancionar y erradicar la violencia contra las mujeres en los ámbitos en que desarrollen sus relaciones interpersonales ; 2009. Available from: http://servicios.infoleg.gob.ar/infoleglnternet/ anexos/150000-154999/152155/norma.htm.

3. Tajer D, Gaba M, Reid G. Impacto de la violencia de género en la salud de las mujeres. Informe final Proyecto ARG 09/016 "Acciones de Protección integral para prevenir, sancionar y erradicar la violencia contra las mujeres", Consejo Nacional de Mujeres, Presidencia de la Nación.; 2011.

4. Essayag S. Del compromiso a la acción: políticas para erradicar la violencia contra las mujeres en América Latina y el Caribe, 2016. Documento de análisis regional. Panamá: Programa de las Naciones Unidas para el Desarrollo; 2017. Available from: http://americalatinagenera.org/newsite/ images/violencia/documentos/DEL_COMPROMISO_A_LA_ACCION_ESP.pdf.

5. Walker L. The Battered Women. New York: Harper and Row Publishers, Inc.; 1979. 